\title{
CARACTERIZAÇÃO MORFOLÓGICA DE Justicia pectoralis JACQ. E $J$. gendarussa BURM. F. (ACANTHACEAE)
}

\author{
Antonio Fernando M. OLIVEIRA, Laíse de Holanda C. ANDRADE
}

RESUMO - Justicia pectoralis e $J$. gendarussa são espécies de interesse medicinal utilizadas popularmente em vários países da América do Sul e Central como analgésicas e antiinflamatórias. As folhas de $J$. pectoralis também são utilizadas por indígenas da Amazônia brasileira e da Venezuela em rapés alucinógenos. $J$. pectoralis e $J$. gendarussa foram caracterizadas morfologicamente sob condições padronizadas de cultivo e investigadas quanto a presença de algum principio alucinógeno. Duas variedades de $J$. pectoralis, J. pectoralis var. stenophylla Leonard e $J$. pectoralis var. A. (indeterminada), foram diferenciadas pelo hábito, forma e número de folhas; $J$. gendarussa difere destas pelo hábito, forma das folhas e coloração das nervuras. $O$ uso de $J$. pectoralis como alucinógena também é discutido.

Palavras-chave: Alucinógeno; Etnobotânica; Morfologia; Justicia gendarussa; Justicia pectoralis.

Morphological Characterization of Justicia pectoralis Jacq. and J. gendarussa Burm. F, (Acanthaceae)

ABSTRACT - Justicia pectoralis and $J$. gendarussa are species of medicinal interest employed in several countries of Central and South America as analgesics and antiinflamatories. The leaves of $J$. pectoralis are also utilized by indigenous of the Brazilian Amazon and Venezuela as hallucinogenic snuffs. J. pectoralis and $J$. gendarussa were morphologically characterized under standardized experimental cultivation and investigated to any evidence of hallucinogenic principle. Two varieties of $J$. pectoralis, J. pectoralis var. stenophylla Leonard and J. pectoralis var. A. (undetermined), were differentiated by the habit, form and number of leaves; $J$, gendarussa differs by the habit, form of the leaves and color of the veins. The use of $J$. pectoralis as hallucinogenic is also discussed.

Key-words: Ethnobotany; Hallucinogen; Morphology; Justicia gendarussa; Justicia pectoralis.

\section{INTRODUÇÃO}

Justicia pectoralis e J. gendarussa têm uso corriqueiro na medicina popular contra dores e inflamações. As espécies ocorrem espontaneamente ou são cultivadas, sendo $J$. pectoralis freqüentemente encontrada no México, Venezuela, Trindad, Cuba, Jamaica, Oeste do Equador, Norte e Nordeste do Brasil; $J$. gendarussa predomina a Oeste da África e Índia e, também, no Nordeste brasileiro (Chagnon et al., 1971;
Morton, 1977; Wasshausen, 1977; van den Berg, 1986; Barros, 1992).

Espécies do gênero Justicia, por apresentarem semelhanças morfológicas entre si, compartilham alguns nomes populares e indicações fitoterápicas. Dentre os seus vários epítetos, "chambá" e "anador" são os mais empregados no Nordeste brasileiro para $J$. pectoralis e $J$. gendarussa (Rizzini, 1948; Chagnon et al., 1971; Barros, 1992).

Diferentes tribos indigenas da Amazônia brasileira e da Venezuela

Departamento de Botânica, Centro de Ciências Biológicas, Universidade Federal de Pernambuco. Av. Prof. Moraes Rego, s/n - Cidade Universitária, Recife - PE, Brasil, CEP:: 50.670.920. 
utilizam rapés alucinogênicos baseados em espécies dos gêneros Virola (Myristicaceae) Anadenanthera (Leg. Mimosoideae). Embora folhas de J. pectoralis já tenham sido identificadas nesses rapés (Chagnon, 1971; Prance, 1972a; 1972b; Schultes, 1979; 1990), a presença de alcalóides nesta espécie ainda é contraditória. Por outro lado, derivados cumarínicos são referidos como seus principais constituintes (Macrae \& Towers, 1984).

Este estudo visou caracterizar morfologicamente duas variedades de $J$. pectoralis e J. gendarussa, desenvolvidas em condições padronizadas de cultivo, além de averiguar a presença de compostos que justifiquem o uso de $J$. pectoralis como alucinógena.

\section{MATERIAL E MÉTODOS}

\subsection{Material estudado}

\subsubsection{Justicia pectoralis Jacq. var. stenophylla Leonard, Herbário UFP $n^{2} 10456$.}

Erva com aproximadamente 29 $\mathrm{cm}$ de altura. Caule ascendente, subcilíndrico a sub-quadrangular, verde com pêlos retrorsos, esbranquiçados, de $0,6 \mathrm{~mm}$ de comprimento, dispostos em linhas verticais; nós inferiores freqüentemente com raizes adventícias; entrenós com $2,0 \mathrm{~cm}$ de comprimento. Folhas opostas, membranáceas, verdes, às vezes com pontuações vináceas; lâmina estreitamente-lanceolada, 1,0-4,0 cm de comprimento, ápice atenuado, base aguda, margem inteira, pilosas em ambas as faces; pêlos com $0,3 \mathrm{~mm}$ de comprimento; pecíolo com $5,0 \mathrm{~mm}$ de comprimento; padrão de venação camptódromo-broquidódromo. Inflorescência racemosa com $5,8 \mathrm{~cm}$ de comprimento, raquis com pêlos glandulosos. Brácteas e bractéolas estreitamente lanceoladas, glabras. Flores sésseis, zigomorfas; cálice verde, 5-lobado, piloso (pêlos glandulosos e não glandulosos); corola tubulosa, branca à lilás, $9,0 \mathrm{~mm}$ de comprimento, tubo reto, lábio superior ereto, triangular, o inferior 3-lobado, externamente pubérulo; estames 2 , inclusos, parcialmente adnatos ao tubo da corola, a parte livre do filete com 3,0 $\mathrm{mm}$ de comprimento; anteras rimosas com 0,5-1,2 $\mathrm{mm}$ de comprimento. Ovário súpero, $1,3 \mathrm{~mm}$ de comprimento, oblongo, bicarpelar, bilocular, 2 óvulos por lóculo; disco glandular na base (nectário), com 0,1 $0,2 \mathrm{~mm}$ de comprimento; estilete terminal, com 5,0 mm de comprimento, pubérulo; Fruto cápsula, clavada, glabra, 1,2-2,3 mm de comprimento. Sementes castanho-avermelhadas, achatadas, com 0,9-1,2 $\mathrm{mm}$ de comprimento (Fig. 1).

\subsubsection{Justicia pectoralis Jacq. var. A, Herbário UFP nº 10897.}

Erva com aproximadamente 30 $\mathrm{cm}$ de comprimento. Caule decumbente, subcilindrico a subquadrangular, verde, com pêlos retrorsos e esbranquiçados, com 0,9 $\mathrm{mm}$ de comprimento, dispostos em 


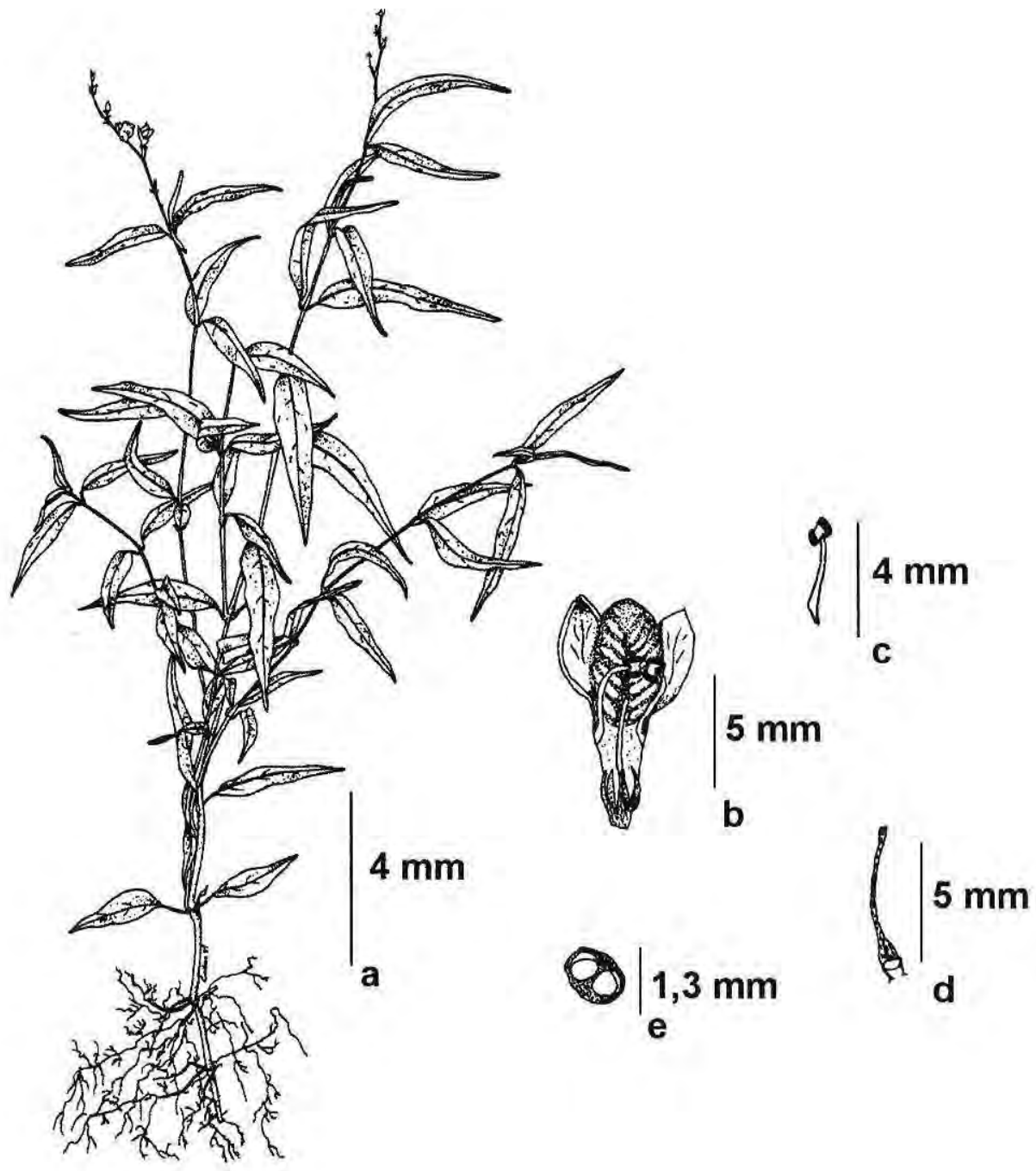

Figura 1. Justicia pectoralis var. stenophylla Leonard: $\mathrm{a}=$ hábito da planta; $\mathrm{b}=$ flor em corte longitudinal; $\mathrm{c}=$ estame; $\mathrm{d}=$ ovário, estilete e estigma; $\mathrm{e}=$ corte transversal do ovário. 
linhas verticais; freqüentemente com raizes adventícias, notadamente nos nós inferiores; entrenós com 2,3 cm de comprimento. Folhas opostas, membranáceas, verdes, às vezes com pigmentação vinácea, mas não tão freqüente quanto na variedade stenophylla; lâmina foliar estreitamente-ovalada, com 2,0-3,5 cm de comprimento, ápice atenuado e base obtusa, margens inteiras, pubescente em ambas as faces, pêlos simples com $0,3 \mathrm{~mm}$ de comprimento; pecíolo com $6,0 \mathrm{~mm}$ de comprimento; padrão de venação camptódromobroquidódromo. Inflorescência racemosa com $6,0 \mathrm{~cm}$ de comprimento. Brácteas e bractéolas estreitamente lanceoladas, glabras. Flores sésseis, zigomorfas; cálice verde, 5-lobado, pubescente (pêlos glandulosos e não glandulosos); corola branca à lilás com $9,0 \mathrm{~mm}$ de comprimento, tubo reto, lábio superior ereto, triangular, o inferior 3-lobado, externamente pubérulo; estames 2 , inclusos, parcialmente adnatos ao tubo da corola, parte livre do filete com 3,2 $\mathrm{mm}$ de comprimento, anteras rimosas, com $0,5-1,3 \mathrm{~mm}$ de comprimento. Ovário súpero com $1,2 \mathrm{~mm}$ de comprimento, oblongo, bicarpelar, bilocular, 2 óvulos por lóculo; disco glandular na base (nectário), com 0,1$1,2 \mathrm{~mm}$ de comprimento; estilete terminal, com 5,2 mm de comprimento, glabro. Fruto cápsula, clavada, pubérula, com 1,3-2,5 $\mathrm{mm}$ de comprimento. Sementes castanhoavermelhadas, achatadas, com 1,0-1,2 mm de comprimento (Fig. 2).

\subsubsection{Justicia gendarussa Burm. F., Herbário UFP nº 08961.}

Subarbusto com aproximadamente $1,0 \mathrm{~m}$ de altura. Caule ereto, cilindrico, lenhoso na base, castanho-vináceo, pêlos simples com $0,9 \mathrm{~mm}$ de comprimento; entrenós com $3,0 \mathrm{~cm}$ de comprimento. Folhas opostas, verde-escuras, membranáceas; lâmina foliar com 1,5-8,0 $\mathrm{mm}$ de comprimento, elíptica, ápice acuminado e base cuneada, de margens inteiras, levemente crenadas, pilosas em ambas as faces, pêlos com $0,4 \mathrm{~mm}$ de comprimento; nervuras vináceas, padrão de venação camptódromo-broquidódroma; pecíolo com $8,0 \mathrm{~mm}$ de comprimento. Inflorescência racemosa com $7,0 \mathrm{~cm}$ de comprimento, pubescente. Brácteas e bractéolas opostas, estreitamente lanceoladas, glabras. Flores sésseis, zigomorfas; cálice castanho, 5-lobado, pubescente; pêlos glandulosos; corola tubulosa branco-rosada com $18,0 \mathrm{~mm}$, tubo reto, lábio superior ereto, triangular, o inferior 3-lobado; estames 2, inclusos, parcialmente adnatos ao tubo da corola, parte livre do filete com 5,0 $\mathrm{mm}$ de comprimento, anteras rimosas, com 0,5-2,0 mm de comprimento. Ovário súpero, com $2,0 \mathrm{~mm}$ de comprimento, vináceo escuro, oblongo, bicarpelar, bilocular, 2 óvulos por lóculo; disco glandular na base (nectário), com 1,0 mm de comprimento; estilete terminal, com $12,0 \mathrm{~mm}$ de comprimento, pubescente. Fruto cápsula, clavada, glabra, com 1,2-3,0 $\mathrm{mm}$ de comprimento. Sementes castanho-avermelhadas, achatadas, com 1,2-1,4 $\mathrm{mm}$ de comprimento (Fig. 3). 


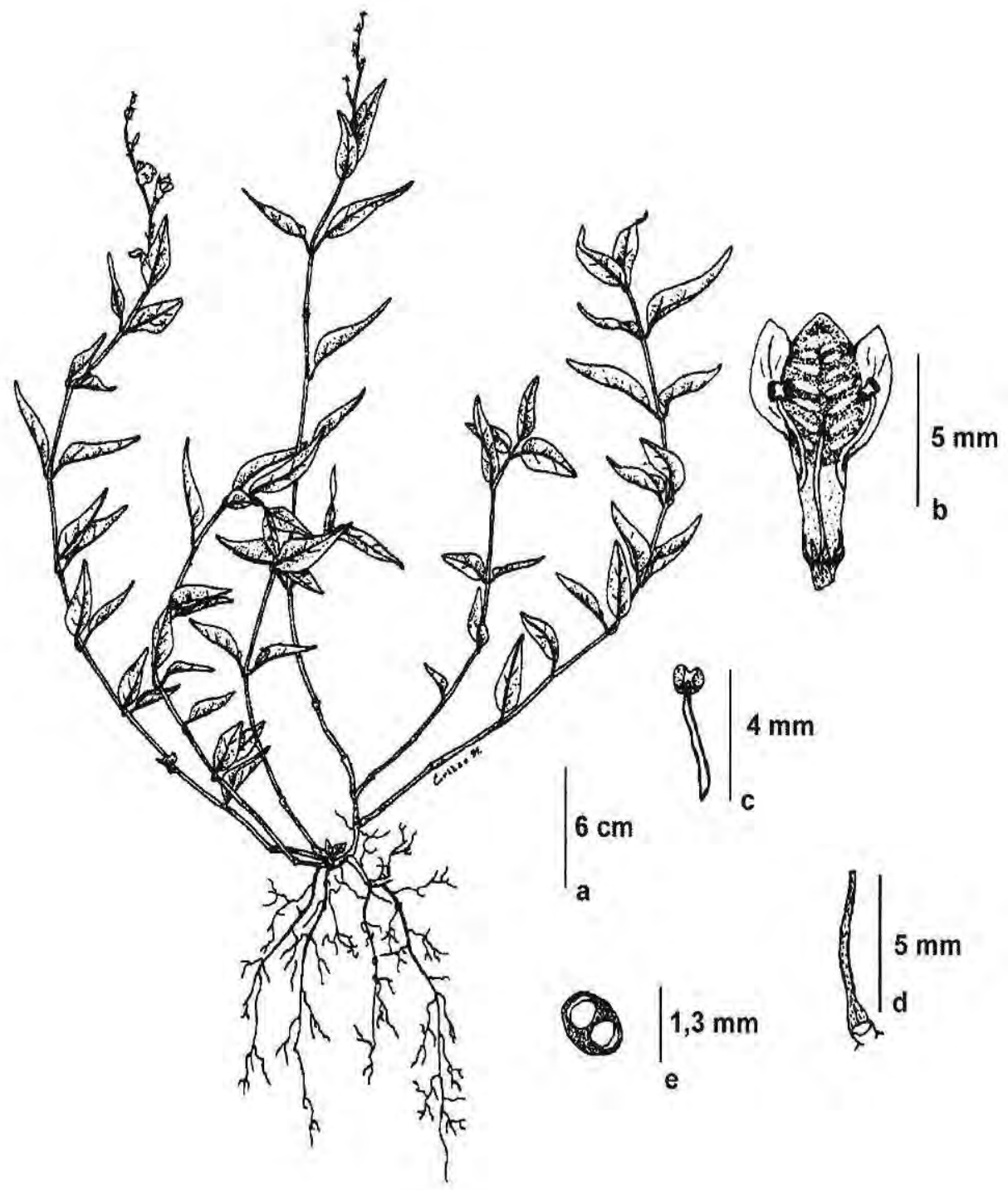

Figura 2. Justicia pectoralis var. A: $\mathrm{a}=$ hábito da planta; $\mathrm{b}=$ flor em corte longitudinal; $\mathrm{c}=$ estame; $\mathrm{d}=$ ovário, estilete e estigma; $\mathrm{e}=$ corte transversal do ovário. 


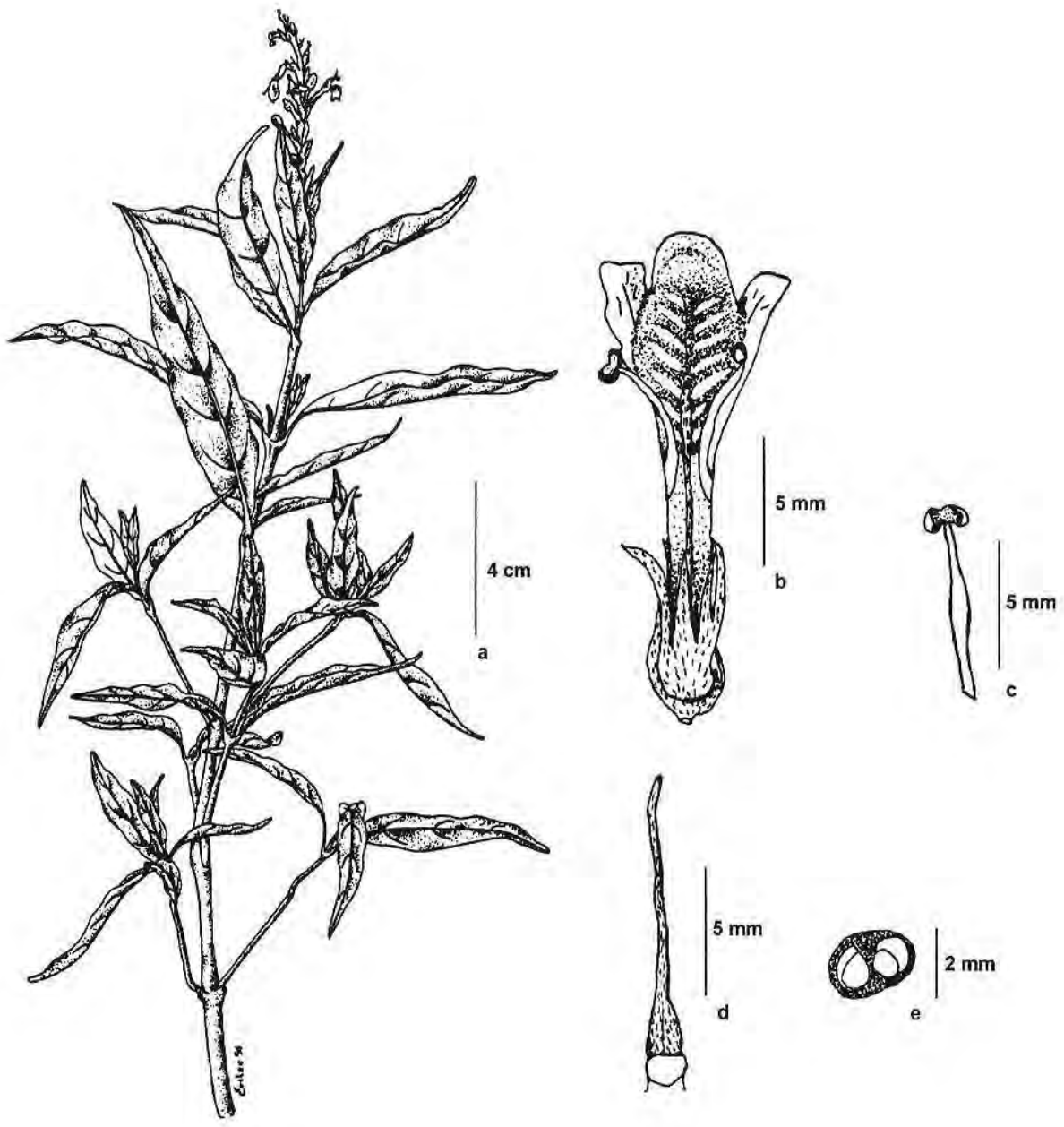

Figura 3. Justicia gendarussa Burm. $\mathrm{F}: \mathrm{a}=$ hábito da planta; $\mathrm{b}=$ flor em corte longitudinal; $\mathrm{c}=$ estame; $\mathrm{d}=$ ovário, estilete e estigma; $\mathrm{e}=$ corte transversal do ovário. 


\subsection{Cultivo}

O estudo desenvolveu-se a partir do plantio de estacas obtidas de plantas matrizes, cultivadas em diferentes áreas no estado de Pernambuco (Brasil). As estacas, sem folhas, foram retiradas da região mediana dos ramos contendo de quatro a cinco pares de gemas foliares (Barros, 1992).

O substrato utilizado para o cultivo consistiu de uma mistura de solo argilo-arenoso acrescido de matéria orgânica (esterco), na proporção de $2,5: 1,5$, respectivamente, acondicionado em sacos de plástico preto com capacidade para $10 \mathrm{~kg}$. Forneceu-se um regime de regas diárias, mantendo-se a umidade próxima a capacidade de campo do solo.

\subsection{Morfologia}

A caracterização morfológica das espécies e variedades foi efetuada segundo metodologia clássica utilizada em trabalhos de taxonomia e a terminologia utilizada foi de acordo com Hickey (1973) e Radford et al. (1974). Para a comparação dos táxones, foram selecionados os seguintes caracteres: hábito das plantas, forma da folha e padrão do sistema de nervação, comprimento dos ramos e entrenós, pilosidade, tipo de inflorescência, coloração e estrutura das flores, frutos e sementes. O material descrito se encontra depositado no Herbário UFP (Recife-PE).

\subsection{Química}

Ensaios cromatográficos para detecção de cumarinas, que constituem os principios ativos de $J$. pectoralis, e de alcalóides, por serem um indicativo do potencial alucinógeno de qualquer espécie vegetal, foram realizados nos três táxones.

Extratos brutos foram obtidos a partir da infusão metanólica de material recém-coletado constituído de folhas e caules. Para a detecção de cumarinas e alcalóides foi realizada cromatografia em camada delgada em placas de gel de sílica G (Merck) e as fases móveis definidas segundo métodos convencionais (Harborne, 1984; Wagner et al., 1983).

\section{RESULTADOS E DISCUSSÃO}

No presente estudo duas variedades de $J$. pectoralis foram diferenciadas pelos caracteres morfológicos, mas apenas uma já era conhecida na literatura: a variedade stenophylla. A variedade não identificada (J. pectoralis var. A) difere da variedade típica pectoralis descrita por Jacquin (1760) pelo menor tamanho da planta, hábito decumbente e lâmina foliar estreitamente-ovalada. Também pode ser distinguida da variedade stenophylla (Leonard, 1958) pelo hábito decumbente, menor número ramos e folhas e pela lâmina foliar estreitamente-ovalada. $\mathrm{O}$ cultivo experimental padronizado permite excluir o efeito ambiental como o causador das diferenças morfológicas observadas entre as duas variedade de J. pectoralis. O hábito subarbustivo é o que mais facilmente distingue $J$. gendarussa de ambas variedades de $J$. 
pectoralis. As principais características morfológicas que diferenciam os três táxones analisados encontram-se na Tabela 1.

Apesar das visiveis variações morfológica entre as duas variedades de $J$. pectoralis, ambas apresentaram 1,2-benzopirona e umbeliferona como constituintes principais, notadamente nas folhas. Quantitativamente, a variedade stenophylla possui um maior teor de cumarinas. Nenhum derivado cumarinico foi detectado nas folhas e caule de $J$. gendarussa. Os testes cromatográficos revelaram ausência de alcalóides nos três táxones examinados, independente do órgão analisado (Tab. 2).

Tabela 1. Principais caracteristicas morfológicas de Justicia pectoralis var. stenophylla, Justicia pectoralis var. A e Justicia gendarussa desenvolvidas em condições padronizadas de cultivo.

Espécies

\begin{tabular}{|c|c|c|c|}
\hline Caracteristica* $^{*}$ & $\begin{array}{c}\text { Justicia pectoralis } \\
\text { var. stenophylla }\end{array}$ & $\begin{array}{c}\text { Justicia pectoralis } \\
\text { var. A }\end{array}$ & $\begin{array}{c}\text { Justicia } \\
\text { gendanussa }\end{array}$ \\
\hline HÁBTO & $\begin{array}{l}\text { herbáceo } \\
\text { cespitoso }\end{array}$ & $\begin{array}{c}\text { herbáceo } \\
\text { decumbente }\end{array}$ & subarbustivo er \\
\hline & $28,5 \mathrm{~cm}$ alt. & $29,3 \mathrm{~cm}$ alt. & $95,5 \mathrm{~cm}$ alt \\
\hline \multicolumn{4}{|l|}{ LÂMINA FOLIAR } \\
\hline Comprimento & $1,0-0,4 \mathrm{~cm}$ & $3,3-2,0 \mathrm{~cm}$ & $8,0-1,5 \mathrm{~cm}$ \\
\hline Forma & $\begin{array}{l}\text { estreitamente- } \\
\text { lanceolada }\end{array}$ & $\begin{array}{l}\text { estreitamente- } \\
\text { ovada }\end{array}$ & $\begin{array}{l}\text { elíptica- } \\
\text { acuminada }\end{array}$ \\
\hline Ápice & atenuado & atenuado & cuneado \\
\hline Base & aguda & obtusa & cuneada \\
\hline
\end{tabular}

FLOR

Corola

\begin{tabular}{lccc}
$\begin{array}{l}\text { Coloração } \\
\text { Dimensão }\end{array}$ & $\begin{array}{c}\text { branca a lilás } \\
\text { Cálice }\end{array}$ & $\begin{array}{c}\text { branca a lilás } \\
9,0 \mathrm{~mm}\end{array}$ & $\begin{array}{c}\text { branca-rosada } \\
18,0 \mathrm{~mm}\end{array}$ \\
$\begin{array}{l}\text { Coloração } \\
\text { Estames }\end{array}$ & verde & verde & castanho \\
Filete & $3,0 \mathrm{~mm}$ & $3,2 \mathrm{~mm}$ & $5,0 \mathrm{~mm}$ \\
Anteras & $0,5-1,2 \mathrm{~mm}$ & $0,5-1,3 \mathrm{~mm}$ & $0,5-2,0 \mathrm{~mm}$ \\
Ovário & & & \\
Coloração & verde & verde & vináceo \\
Dimensão & $1,3 \mathrm{~mm}$ & $1,2 \mathrm{~mm}$ & $2,0 \mathrm{~mm}$ \\
\hline
\end{tabular}

* apenas estăo listados os caracteres morfológicos que diferenciam os táxones. 
A presença de cumarinas em $J$. pectoralis já foi referida por vários autores (Macrae \& Towers, 1984; Mills et al., 1986; De Vries et al., 1988; Barros, 1992; Lino et al., 1997) sugerindo serem tais substâncias as principais responsáveis pela sua atividade fitoterápica. A ausência de alcalóides em $J$. pectoralis, por outro lado, foi mencionada por Macrae \& Towers (1984) e por Melo \& Andrade (1989), apesar de Duke (1987) revelar a presença de pequenas quantidades de triptaminas em $J$. pectoralis, as quais, segundo o autor, seria uma forte evidência de princípio alucinógeno nesta espécie.

Os ensaios fitoquímicos com $J$. gendarussa confirmam os resultados de Govindachari et al. (1969) sobre a ausência de alcalóides e cumarinas nesta planta. Deste modo, apesar desta espécie ser também empregada popularmente como analgésica e antiinflamatória, sua atividade fitoterápica é devida a outros princípios diferentes das cumarinas, provavelmente flavonóides C- glicosilados (Oliveira, 1995)

Nenhuma das substância identificadas em $J$, pectoralis, justifica - seu uso como alucinógena. Provavelmente, o odor agradável das cumarinas ainda é a causa principal da utilização desta espécie em rapés alucinogênicos por tribos indígenas da Amazônia brasileira e da Venezuela.

\section{AGRADECIMENTOS}

Os autores agradecem o auxílio da $\mathrm{Dr}^{\mathrm{a}}$. Sheila Regina Profície do Jardim Botânico do Rio de Janeiro (Brasil) pela identificação das espécies e ao Conselho Nacional de Desenvolvimento Científico e Tecnológico (CNPq) pelo suporte financeiro.

\section{Bibliografia citada}

Barros, R.F.M. 1992. Efeito da radiação solar sobre o crescimento e produçãode cumarinas em Justicia pectoralis var. stenophylla Leonard. Dissertação de Mestrado. Universidade Federal Rural de Pernambuco, Recife. 143p.

Tabela 2. Metabólitos secundários investigados através de cromatografia em camada delgada em Justicia pectoralis var. stenophylla, J. pectoralis var. A e $J$. gendarussa desenvolvidas em condições padronizadas de cultivo.

Espécies

Metabólitos Justicia pectoralis var. stenophylla
Justicia pectoralis var. A
Justicia

gendarussa

$\begin{array}{lllllllll}f & c & f & c & f & c\end{array}$

Alcalóides

Cumarinas

$+++\quad++$

$+$

$(-)$ ausente $(+)$ fraco $(++)$ médio $(+++)$ forte

$f=$ folha $c=$ caule 
Chagnon, N.A; Le Quesne, P; Cook, J.M. 1971. Yanomame halucinogens: anthropological, botanical and chemical findings. Current Anthropol., 12: 72-74.

De Vries, J.X; Tauscher, B; Joshi, B.S. 1988. Constituents of Justicia pectoralisJacq. Gas chromatography/mass spectrometry of simple coumarins, 3-phenilpropionic acid and their hydroxy and methoxy derivatives acid and their hydroxy and methoxy derivatives. Biomed. Environ. Mass. Spectrom., 15: 413-417.

Duke, J.A. 1987. Handbook of medicinal herbs. Florida, CRC Press. 677p.

Govindachari, T.R; Jadhav, S.J; Joshi, B.S. 1969. Chemical investigation of some indian plants: Part IV. Indian J. Chem., 7: 308-310.

Harborne, J.B. 1984. Phytochemical methods. 2.rd. London, Chapman \& Hall. 288p.

Hickey, L.J. 1973. Classification of the architecture of dicotyledonous leaves. Amer. J. Bot., 60: 17-33.

Jacquin, N.J. 1760. Sertum botanicum: enumeratio systematica plantarum, 5: 11.

Leonard, E.C. 1958. The Acanthaceae of Colombia, III. Contributions from the United States National Herbarium, 31: 613-615.

Lino, C.S; Taveira, M.L; Viana, G.S.B; Matos, F.J.A. 1997. Analgesic and anti-inflammatory activities of Justicia pectoralis Jacq and its mais constituents: coumarin and umbelliferone. Phytother: Res., 11: 211-215.

Macrae, W D; Towers, G.H.N. 1984. Justicia pectoralis: a study of the basis for it's use a hallucinogenic snuff ingredient. $J$. Ethnopharm., 12: 93-111.

Melo, R.F; Andrade, L.H.C. 1989. Contribuição ao estudo farmacognóstico do chambá Justicia pectoralis Jacq. (Acanthaceae), Biol. Bras., 1:195-207.

Mills, J; Pascoe, K.O; Chambers, J. 1986. Preliminary investigations of the wound healing properties of a Jamaica folk plant (Justicia pectoralis). West Indian Med. J., 35: 190-193.
Morton, J.F. 1977. Some folk-medicine plants of Central American markets. Quat. J. Crude Drug Research., 15: 165-162.

Oliveira, A.F.M. 1995. Caracterização de Acanthaceae medicinais conhecidas como anador no Nordeste do Brasil. Dissertação de Mestrado, Universidade Federal de Pernambuco, Recife, Pernambuco, 125p.

Prance, G.T. 1972a. An ethnobotanical comparison of four tribes of amazonian indians. Acta Amazonica, 2: 7-28.

Prance G.T. 1972b Ethnobotanical notes from Amazonian Brazil. Economic Botany, 26: 221-237.

Radford, A.E; Dickison, W.C; Massey, J.R. 1974. Vascular plant systematics. N e w York, Harper \& Tow Pulblishers, 891p.

Rizzini, C.T. 1948. Contribuição ao conhecimento da tribo Justicieae (Acanthaceae). Arq. Jard Bot., 9: 37-69.

Schultes, R.E. 1979. Evolution of the identification of the Myristicaceous hallucinogens of South America. J. Ethnopharm., l: 211-239.

Schultes, R.E. 1990. De plantis toxicaris e Mundo Novo Tropicale commentationes XXXVI Justicia pectoralis (Acanthaceae) as a source of an hallucinogenic snuff. Economic Botany, 44: 61-70.

van den Berg, M.E. 1986. Ethnobotany of a traditional ablution in Pará, Brazil. Boletim do Museu Paraense Emilio Goeldi, Botânica, 2: 213-218.

Wagner, H; Bladt, S; Zgainski, E.M. 1983. Drogennanalyse. Berlim, Springer Verlag, $164 \mathrm{p}$

Wasshausen, D.C. 1977. New taxa of Acanthaceae from the Rio Palenque Science Center in Equador. Selbyana, 2: 14-22. 